Der Chemie-Preis 2009 wurde Herrn Philip Tinnefeld, München, in Anerkennung seiner grundlegenden Arbeiten zur Weiterentwicklung der optischen Einzelmolekülspektroskopie und ihrer Anwendungen auf biomolekulare Wechselwirkungen verliehen.

\title{
Fluoreszenzmikroskopie „bottom-up“: Von Einzelmolekülen zur Superauflösung
}

\section{Philip Tinnefeld}

Die räumliche Auflösungsfähigkeit eines optischen Mikroskops ist durch die Wellenlänge des Lichts begrenzt. So können mit einem herkömmlichen Mikroskop Strukturen, die kleiner als 200 bis 300 Nanometer also Millionstel Millimeter - sind, nicht mehr eindeutig voneinander abgegrenzt werden. Diese fundamentale physikalische Grenze der Lichtmikroskopie wird von der Wellennatur des Lichts und der damit einhergehenden Beugung verursacht: Licht einer bestimmten Wellenlänge, das von einer Linse fokussiert wird, kann keine Objekte voneinander trennen, die näher als ungefähr die halbe Wellenlänge des Lichts voneinander entfernt sind.

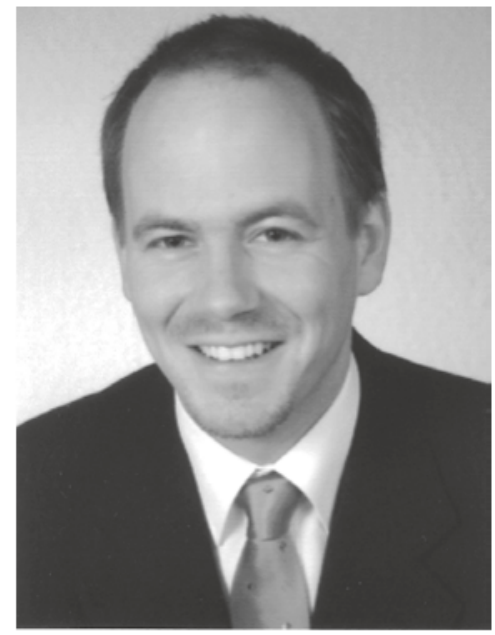

Philip Tinnefeld, Professor für Biophysik an der Ludwig-MaximiliansUniversität München, Träger des Chemie-Preises 2009

Diese seit etwa 140 Jahren bekannte Grenze galt allgemein als mit optischen Fernfeldmethoden unüberwindbar. Dem gegenüber steht das Interesse der Wissenschaftler an Strukturen von der Größe einzelner Moleküle und biomolekularer Komplexe. Dynamisch interagierende Zusammenschlüsse von Biomolekülen mit einer Größe von 10 bis $100 \mathrm{~nm}$ führen die fundamentalen molekularen Prozesse aus, die in ihrer Summe das Leben ausmachen. Eine höhere Auflösung ist zwar mit elektronenmikroskopischen Verfahren möglich, doch diese sind aufwändig und besitzen gewisse Nachteile - zum Beispiel erlauben sie keine Analyse lebender Strukturen. 
A

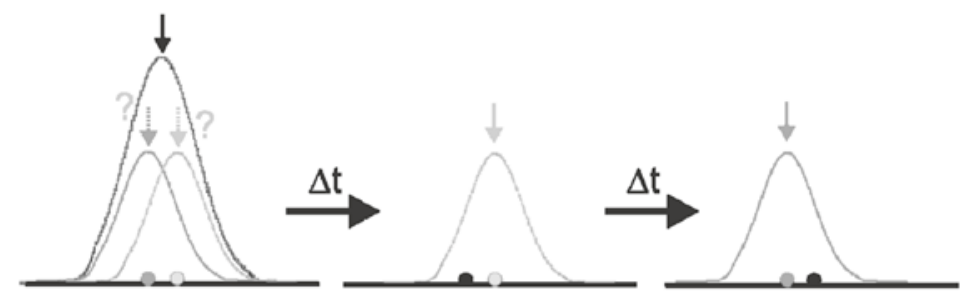

B

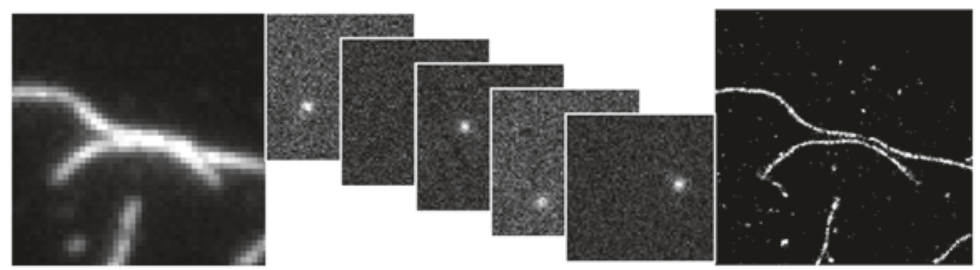

Abbildung 1: A: Schema der Fluoreszenz-Nanoskopie, die durch die Lokalisation einzelner Farbstoffmoleküle und ihrer zeitlichen Trennung Superauflösung erreicht. Wenn beide Moleküle gleichzeitig leuchten und ihre Anzahl und jeweilige Helligkeit nicht bekannt ist, dann ist die Strukturinformation aufgrund der Beugung verloren. Durch zeitliche Trennung ihrer Fluoreszenz können die beiden Moleküle sehr genau in der Mitte der Abbildungsfunktion lokalisiert werden. B: Beispiel der Blink-Mikroskopie: Beugungsbegrenztes Bild von Aktinfilamenten auf Glasoberflächen (links); einige Bilder einer Zeitserie, die die Emission einzelner blinkender Moleküle zeigt (Mitte), und das aus der sukzessiven Lokalisation der einzelnen Moleküle rekonstruierte Superauflösungsbild (rechts).

Ein erster Durchbruch zur Entwicklung einer Methode, die die Beugungsgrenze im Fernfeld überwindet, wurde mit der Stimulated Emission Depletion Mikroskopie bereits 1994 von Wichmann und Hell vorgestellt und einige Jahre später realisiert. [1] Hierbei werden die fluoreszierenden Farbstoffmoleküle in einem konfokalen Mikroskop angeregt. Der Anregungsfokus hat dabei einen beugungsbegrenzten Durchmesser von ca. $250 \mathrm{~nm}$. Durch Überlagern mit einem zweiten längerwelligen Laser, der aufgrund von destruktiver Interferenz ein Intensitätsminimum in der Mitte des Fokus hat, werden Moleküle im äußeren Fokusbereich über stimulierte Emission abgeregt, was aufgrund der Nichtlinearität der Abregung zu einer nichtbeugungsbegrenzten Auflösung führt.

Mittlerweile hat sich hieraus ein neues Gebiet mit vielfältigen Ansätzen zur Auflösungserhöhung entwickelt $(\Rightarrow$ Nanoskopie oder FernfeldSuperauflösungsmikroskopie). Gemeinsam ist diesen Ansätzen, dass sie das Schalten der Farbstoffe zwischen einem leuchtenden und einem dunklen Zustand aufweisen müssen, damit die Fluoreszenz der Farbstoffe getrennt werden und die Moleküle sequenziell lokalisiert werden können. Hier ha- 
ben sich in letzter Zeit zwei Ansätze durchgesetzt, zum einen das gezielte Auslesen, bei dem der Ort durch die Anregungsgeometrie vorgegeben ist und geschaut wird, ob sich an diesem Ort fluoreszierende Moleküle befinden (z. B. bei der STED Mikroskopie), zum anderen das Auslesen der Positionen einzelner Farbstoffmoleküle in einer Probe, wobei die Farbstoffe stochastisch zwischen einem An- und einem Auszustand hin- und hergeschaltet werden. Die letztere Methode hat sich aufgrund ihrer instrumentellen Einfachheit - es wird lediglich ein Weitfeld-Mikroskop mit hochempfindlicher CCD-Kamera benötigt - weltweit in viele Laboratorien ausgebreitet. Während also ein Großteil der Farbstoffmoleküle in einem Dunkelzustand präpariert wird, kann die Population der leuchtenden Moleküle, die so verdünnt vorliegt, dass die Emission einzelner Moleküle nicht mehr überlappt und abgegrenzte beugungslimitierte Punkte detektiert werden, in der Mitte dieser Abbildungsfunktionen zum Beispiel mit Hilfe einer Gaußanpassung hochpräzise bestimmt werden. Diese Ortsbestimmung hat je nach Anzahl der detektierten Photonen eine Genauigkeit von wenigen Nanometern. Die Orte der Lokalisationen werden anschließend in einem neuen 2-D Bild histogrammiert und ergeben farbcodiert das rekonstruierte, superaufgelöste Bild. Dieser Prozess ist in Abbildung 1 anhand einzelner Aktinfilamente dargestellt, die auf einem Deckglas polymerisiert vorliegen.

Ein zentraler Punkt, in dem sich die verschiedenen Ansätze unterscheiden, die das stochastische Schalten und Positionsauslesen benutzen, sind die Art und Weise, wie ein Großteil der Farbstoffmoleküle in einem Auszustand präpariert werden kann. Hierzu bieten sich zum Einen photochrome Farbstoffe an, die mit Hilfe zweier Anregungswellenlängen an- und ausgeschaltet werden können. Außerdem spielen in diesem Bereich photoschaltbare, fluoreszierende Proteine wie Derivate des Grün-Fluoreszierenden-Proteins (GFP) eine Rolle, da sie vor allem für die Markierung in lebenden Zellen geeignet sind. Aus photophysikalischer Sicht sind die verwendeten photochromen Farbstoffe aber alles andere als ideal und werden schnell photozerstört. Auch ist die Schaltung mit häufig kürzerwelligem Licht nicht perfekt, wenig reproduzierbar und führt zu zusätzlicher Photozerstörung. Wir haben in den letzten Jahren einen neuartigen Weg gefunden, um diese Photozerstörung zu reduzieren und generische Auszustände, die jeder Fluoreszenzfarbstoff aufweist, für die Nanoskopie zu nutzen [2]. Dazu werden transiente Zustände der Farbstoffe, wie Triplett- und Radikalzustände, mit Hilfe von Elektronentransferreaktionen depopuliert. 
Es ist allgemein akzeptiert, dass langlebige Zustände wie Triplettzustände entscheidende Zwischenstufen auf dem Weg zur Photozerstörung organischer Farbstoffe sind. Um die Triplettzustände rasch zu entvölkern, haben wir vorgeschlagen, redox-aktive Substanzen, d.h. Reduktions- und Oxidationsmittel einzusetzen. Diese reagieren in einer Elektronentransferreaktion mit dem angeregten Triplettzustand des Farbstoffs und überführen ihn in das entsprechende Radikalion (siehe Schema in Abbildung 2). Da diese Radikalionen aber ebenfalls potentiell reaktiv sind und somit über Nebenreaktionen zu nichtfluoreszierenden Photoprodukten führen können, müssen auch die Radikalzustände schnell entvölkert werden. Dies erfolgt dadurch, dass Reduktions- und Oxidationsmittel in einem reduzierenden und oxidierenden System (ROXS) gleichzeitig eingesetzt werden. Sobald sich ein Radikalion bildet, reagiert es rasch über die komplementäre Redoxreaktion

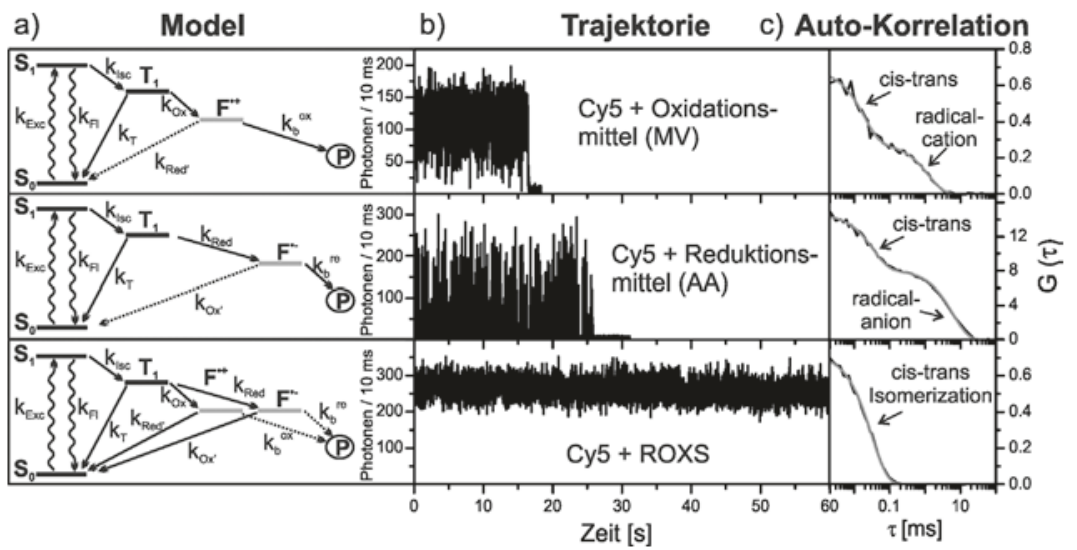

Abbildung 2: Das Konzept des reduzierenden und oxidierenden Systems (ROXS): (a) Zu Grunde liegendes Energieschema, das beschreibt, welche Reaktionswege in Anwesenheit eines Oxidationsmittels (oben), eines Reduktionsmittels (Mitte) bzw. von beidem (unten) möglich sind (S: Singulett, T: Triplett, F: Radikal, P: photozerstört) (b) Fluoreszenzzeitspuren (Trajektorien) einzelner Cy5 Farbstoffmoleküle, in Anwesenheit des Oxidationsmittels Methylviologen (oben), des Reduktionsmittels Askorbinsäure (Mitte) oder von beidem gleichzeitig (unten). (c) Die Autokorrelationsfunktion des Fluoreszenzsignals zeigt die Zeitskalen von Intensitätsfluktuationen. In Anwesenheit von ROXS wird nur die Intensitätsfluktuation aufgrund einer cis-trans Isomerisierung des Farbstoffs detektiert. Die Lebensdauer aller anderen transienten Zustände ist massiv verkürzt und wird daher nicht mehr detektiert. 
wieder in den Grundzustand und steht wieder für Fluoreszenzzyklen zur Verfügung. Dieses Prinzip ist in Abbildung 2 anhand des häufig verwendeten Cyanin-Farbstoffs Cy5 dargestellt. In Anwesenheit eines Oxidationsmittels zeigt der Farbstoff schnelles sogenanntes „Blinken“. Die mit Hilfe einer Autokorrelation (Abbildung 2c) bestimmten Auszustände im Millisekundenbereich können der Bildung eines Radikalkations zugeordnet werden. Die Photostabilität ist unter diesen Bedingungen stark eingeschränkt: Das Molekül wird nach ca. 16 s irreversibel zerstört, wie aus dem schlagartigen Verschwinden des Fluoreszenzsignals hervorgeht (Abb. 2a, oben). Eine weitere Intensitätsfluktuation, die in der Autokorrelation auftaucht, kann der für diesen Farbstoff typischen cis-trans Isomerisierung zugeordnet werden (Abb. 2c). Wird dem Farbstoff alternativ ein Reduktionsmittel zugesetzt, treten ähnliche Auszustände auf, die aber mit einer Lebensdauer von bis zu $60 \mathrm{~ms}$ etwas länger lebig sind. Dies wird auf die wiederholte Bildung von Radikalanionen zurückgeführt, die in diesem Fall nach ca. 26 s ebenfalls zur Photozerstörung führen. Werden jetzt Reduktions- und Oxidationsmittel gleichzeitig eingesetzt, verschwinden die Radikalzustände in der Intensitätsspur vollständig, und das Molekül leuchtet gleichmäßig und stabil (Abb. 2b, unten). Die Autokorrelation zeigt ferner, dass bis auf cis-trans-Isomerisierung keine weiteren Auszustände mit einer Lebensdauer $>1 \mu$ auftreten.

Die Reduktions- und Oxidationsmittel reagieren dabei gewöhnlich nicht miteinander, da die einzelnen Reaktionsschritte letztendlich durch die Anregungsenergie des Farbstoffes angetrieben werden und aus chemischer Sicht der Photozyklus eine Photokatalyse mit dem Farbstoff als Katalysator darstellt. Dieses Konzept der Photostabilisierung organischer Farbstoffe ist allgemein anwendbar und klärt auch den Ursprung und die Eigenschaften des oft unwillkommenen Blinkens einzelner Quantenemitter.

Neben der zum Beispiel für die STED-Mikoskopie benötigten höheren Photostabilität, bietet das über das Blinken Gelernte einen neuen Schaltmechanismus für die Superauflösungsmikroskopie: Radikalzustände, die langlebig genug sind, können dafür genutzt werden, einen Großteil der Farbstoffmoleküle auszuschalten und die verbliebene Subpopulation auszulesen. Dies ist schließlich in besonders kontrollierter Form gelungen, indem ausgesprochen elektronenaffine Farbstoffe wie Oxazine eingesetzt wurden [3]. Diese Farbstoffe besitzen einen so stabilen Radikalanionenzustand, dass es vergleichsweise starker Oxidationsmittel bedarf, um sie wieder anzuschalten. In Abwesenheit eines Oxidationsmittels können diese Farbstoffe sogar dauerhaft ausgeschaltet werden (siehe rote und gelbe Punkte in Abbildung 3). 


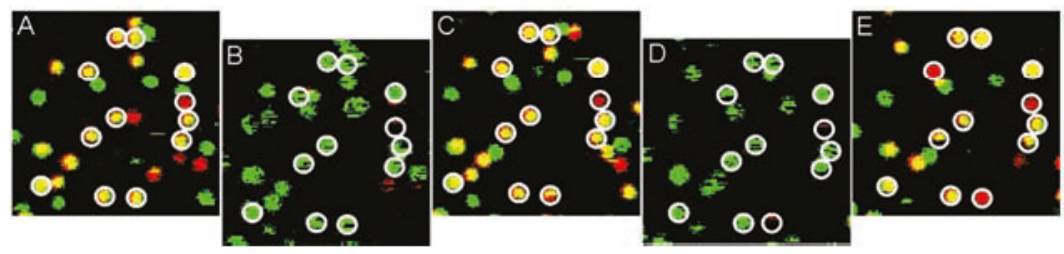

Abbildung 3: Fluoreszenzbilder einzelner Moleküle auf einer Glasoberfläche. Die rot dargestellten Punkte repräsentieren den elektronaffinen Farbstoff AT'TO655. Der grüne Farbstoff Cy3B wurde mit angebracht, um die gleichen Stellen auf der Oberfläche zu identifizieren, wenn ATTO655 in einer reduzierenden Umgebung nicht leuchtet (B, D). Gelbe Punkte repräsentieren Kolokalisierungen der beiden Farbstoffe im Falschfarbenbild. Die Bedingungen wurden zwischen normalem Phosphat-Puffer (A, C, E) und reduzierendem Puffer (mit Askorbinsäure, Sauerstoff entfernt)(B, D) alterniert. Die roten Farbstoffe werden vorübergehend in einen metastabilen reduzierten Zustand geschaltet.

Mit diesen Farbstoffen gelang diese superaufösende „Blink-Mikroskopie“ sogar in Anwesenheit von Sauerstoff und in Zellen. Ein so erhaltenes Bild ist beispielsweise in Abbildung 1 gezeigt.

Künftig sollen die verwendeten Fluoreszenzfarbstoffe gezielt den Umgebungsbedingungen in lebenden Zellen angepasst werden, und Strukturen von biomolekularen Komplexen in Zellen sollen aufgelöst werden. Generell steht aber schon fest, dass die neuen Techniken der Nanoskopie bisher unerreichte Einblicke in die Struktur biologischer Zellen ermöglichen.

\section{Literatur}

[1] S. W. Hell, Far-field optical nanoscopy, Science 2007, 316, 1153.

[2] J. Vogelsang, R. Kasper, C. Steinhauer, B. Person, M. Heilemann, M. Sauer, P. Tinnefeld, $A$ reducing and oxidizing system minimizes photobleaching and blinking of fluorescent dyes, Angew Chem Int Ed 2008, 47, 5465; C. Steinhauer, C. Forthmann, J. Vogelsang, P. Tinnefeld, Superresolution microscopy on the basis of engineered dark states, J. Am. Chem. Soc. 2008, 130, 16840.

[3] J. Vogelsang, T. Cordes, C. Forthmann, C. Steinhauer, P. Tinnefeld, Controlling the fluorescence of ordinary oxazine dyes for single-molecule switching and superresolution microscopy, Proc Natl Acad Sci US A 2009, 106, 8107. 